\title{
A globally distributed Syndiniales parasite dominates the Southern Ocean micro-eukaryote community near the sea-ice edge
}

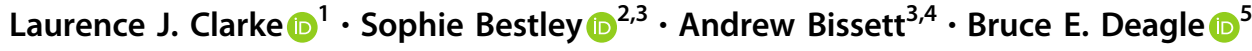

Received: 13 July 2018 / Revised: 4 October 2018 / Accepted: 9 October 2018 / Published online: 26 October 2018

(c) International Society for Microbial Ecology 2018

\begin{abstract}
Syndiniales (Dinophyceae, Alveolata) are a diverse parasitic group common in all marine environments, but their ecological role remains poorly understood. Here we show an unprecedented dominance of a single Syndiniales group I operational taxonomic unit (OTU) across $3000 \mathrm{~km}$ of Southern Ocean transects near the sea-ice edge. This super-abundant OTU consistently represented $>20 \%$, and in some locations $>50 \%$, of eukaryote $18 \mathrm{~S}$ rDNA sequences. Identical $18 \mathrm{~S}$ V4 sequences have been isolated from seven Northern Hemisphere locations, and the OTU's putative V9 rDNA sequence was detected at every station of the global Tara Oceans voyage. Although Syndiniales taxa display some host specificity, our identification of candidate Southern Ocean hosts suggests this OTU associates with distinct phyla in different parts of the world. Our results indicate Syndiniales are key players in surface waters near the vast and dynamic sea-ice edge in the world's most biologically productive ocean.
\end{abstract}

\section{Introduction}

The parasitic Syndiniales group often dominate marine $18 \mathrm{~S}$ ribosomal DNA (rDNA) surveys in both diversity and sequence abundance $[1,2]$, particularly in the smallest $(<5$ $\mu \mathrm{m})$ size fraction. All described Syndiniales obligately kill their host [3], including other protists (dinoflagellates, cercozoans, radiolarians) and metazoans (copepods, fish eggs), with free-living dinospores released following host death [3,

Electronic supplementary material The online version of this article (https://doi.org/10.1038/s41396-018-0306-7) contains supplementary material, which is available to authorized users.

Laurence J. Clarke

laurence.clarke@utas.edu.au

1 Antarctic Climate and Ecosystems Cooperative Research Centre, University of Tasmania, Hobart, Tasmania 7001, Australia

2 Institute for Marine and Antarctic Studies, University of Tasmania, Hobart, Tasmania 7001, Australia

3 Commonwealth Scientific and Industrial Research Organisation, Oceans and Atmosphere, Hobart, Tasmania 7004, Australia

4 Commonwealth Scientific and Industrial Research Organisation, Environomics FSP, Hobart, Tasmania 7004, Australia

5 Australian Antarctic Division, Channel Highway, Kingston, Tasmania 7050, Australia
4]. Formally described Syndiniales taxa display some host specificity, with genetically distinct members of Syndiniales group II related to the succession of bloom-forming dinoflagellates [5]. Syndiniales, therefore, can potentially influence plankton population dynamics and biogeochemical cycling $[6,7]$. Here we show an unprecedented dominance of a single Syndiniales group I operational taxonomic unit (OTU) across $3000 \mathrm{~km}$ of Southern Ocean survey transects in productive surface waters near the sea-ice edge.

\section{Results}

\section{Globally distributed Syndiniales group I OTU dominates samples near sea-ice edge}

Our data set contained 4.25 million 18S V4 rRNA gene sequences representing 1566 (97\%) OTUs across 155 sizefractionated surface seawater samples. Southern Ocean eukaryotic plankton communities showed similar OTU richness and phylogenetic diversity to temperate and tropical regions, with the Syndiniales group representing a comparable proportion of OTUs as found in the Tara Oceans study ( $\sim 15$ vs. $\sim 12 \%$ for Tara Oceans [1]). However, we found one OTU assigned to Syndiniales group I (clade 1, ref. [3], hereafter SG1-OTU) dominated samples near the sea-ice edge (Fig. 1a). In fact, the SG1-OTU 

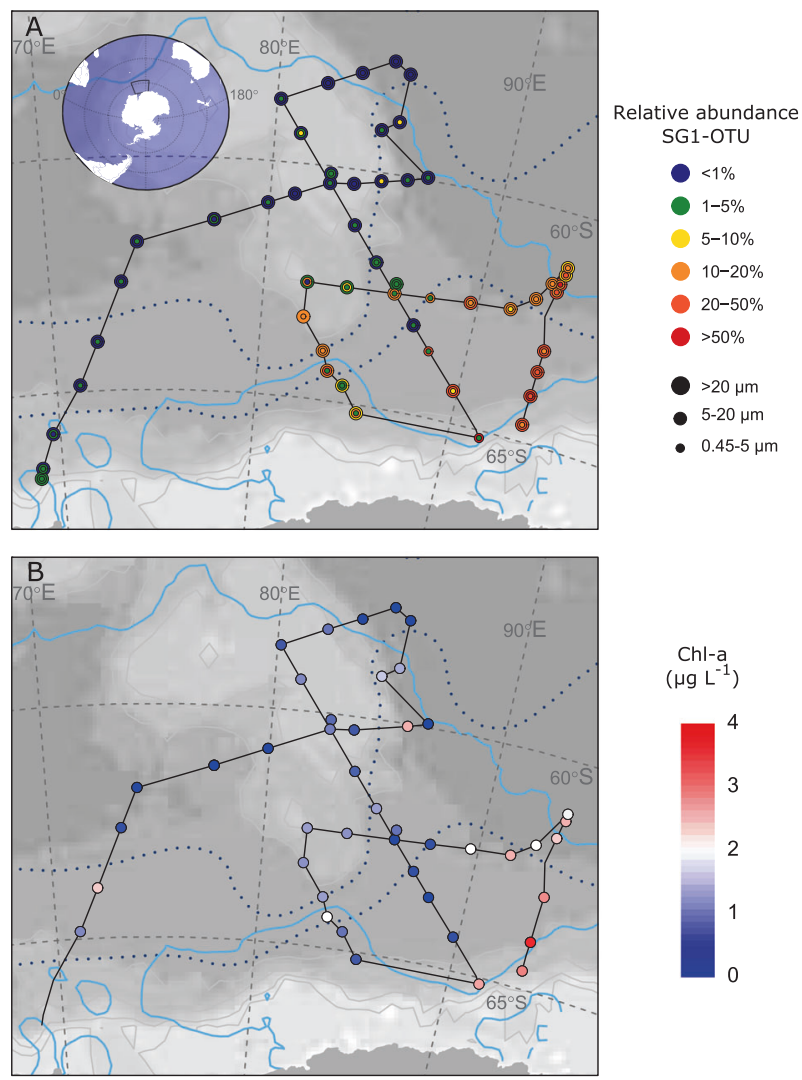

$\left(\mu \mathrm{L}^{-1}\right)$
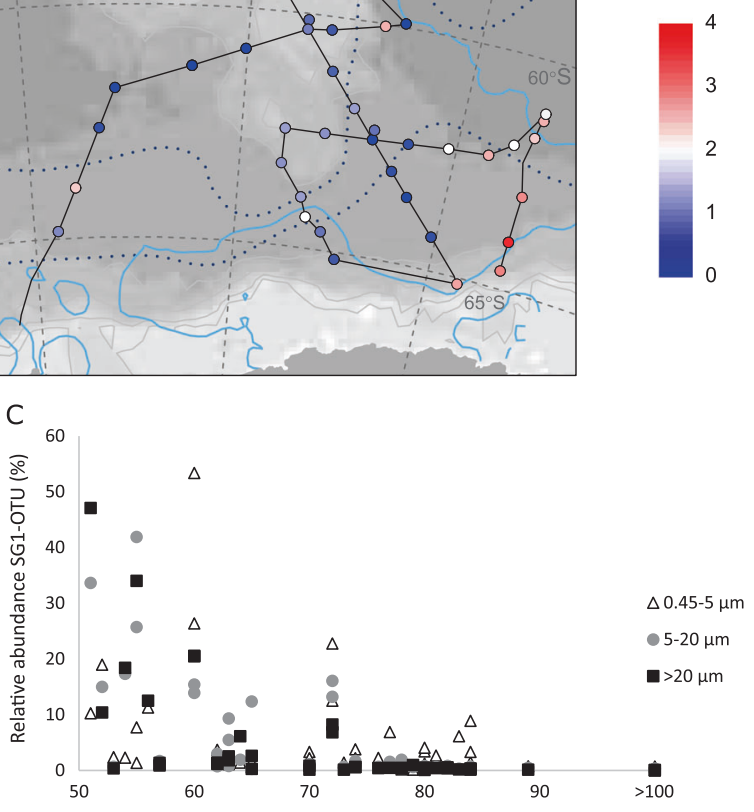

Days since melt

Fig. 1 Syndiniales group I OTU (SG1-OTU) dominates samples near sea-ice edge. a The concentric circles show the relative abundance of SG1-OTU in three surface plankton size fractions $(0.45-5,5-20$, and $>20 \mu \mathrm{m}$ ) south of the Kerguelen Plateau region of the Southern Ocean. b Surface chlorophyll $a$ in the same region. Both SG1-OTU relative abundance and surface chlorophyll were highest in the easternmost transect. Upper and lower blue lines are sea ice in November and January, respectively. Historical positions of southern Antarctic Circumpolar Current (ACC) front and Southern Boundary of the ACC are shown as dotted lines. $\mathbf{c}$ Relative abundance of SG1-OTU in each size fraction in relation to days since sea-ice melt. Only the period following 50 days since melt is shown to highlight the greater relative abundance in the pico-nanoplankton compared to the other size fractions following 80 days since melt

represented more than $20 \%$ of $18 \mathrm{~S}$ sequencing reads in 21 samples $(5,8$, and 8 samples from the pico-nanoplankton $(0.45-5 \mu \mathrm{m})$, nanoplankton $(5-20 \mu \mathrm{m})$ and microplankton $(>20 \mu \mathrm{m})$ fractions, respectively), and in three of those samples this OTU represented more than half the reads (1 and 2 samples from the pico-nanoplankton and nanoplankton, respectively). OTUs representing autotrophs (Fragilariopsis and Phaeocystis) or metazoan copepods were the only other OTUs to represent $>50 \%$ of reads in a sample.

Minimum entropy decomposition (MED [8]) showed the majority of reads (76.8\%) assigned to the SG1-OTU represent a single MED oligotype, indicating that OTU clustering was not concealing multiple abundant oligotypes. While this is the first Southern Hemisphere record of the SG1-OTU's modal sequence, identical V4 sequences have been recorded from seven Northern Hemisphere locations (Supplementary Fig. 1), including radiolarian-associated sequences from a Norwegian fjord [9].

The putative V9 sequence derived from full-length $18 \mathrm{~S}$ sequences in the NCBI database with $100 \%$ identity to the SG1-OTU V4 region was in the top $0.1 \%$ most abundant metabarcodes in the Tara Oceans V9 rDNA database; being present (albeit at relative abundances $<1 \%$ ) at all 47 stations (Supplementary Fig. 1). In total, $95.8 \%$ of reads were in the pico- and nanoplankton size fractions $(0.8-20 \mu \mathrm{m})$. Hence, the SG1-OTU is globally distributed, but typically present at low abundance.

\section{Cercozoan OTU abundance and environment influence SG1-OTU abundance}

Syndiniales with similar $18 \mathrm{~S}$ sequences tend to infect hosts within a given taxonomic class [3,9]. Given the identical $18 \mathrm{~S}$ V4 radiolarian-associated sequence, and that Syndiniales group I (clade 1) have only been isolated from radiolarians [9], we expected the Southern Ocean SG1-OTU to be associated with radiolarians.

No radiolarians showed a strong positive association with the SG1-OTU (see Supplementary Materials and Methods). However, the relative abundance of a cercozoan OTU (Rhizaria) showed a strong positive correlation in both size fractions where the parasite is likely to be associated with its host (i.e., nano- and microplankton, Supplementary Fig. 2). This OTU was assigned to the sea-ice-associated Cryothecomonas sp. [10], consistent with higher SG1-OTU abundance near the ice edge (Fig. 1a).

Models relating SG1-OTU relative abundance to Cryothecomonas OTU relative abundance had stronger support (lower AIC values) than models containing only environmental variables (surface chlorophyll $a$ and/or salinity, Supplementary Table 1; likelihood ratio test (LRT) $p$-values $<0.0001)$. However, combining Cryothecomonas OTU relative abundance with chlorophyll $a$ and/or salinity achieved the best fit (Supplementary Table 1; LRT $p$-values $<0.002)$. As well as positive associations with the Cryothecomonas OTU in both the nano- $(F=7.0, p=0.012)$ 
and microplankton $(F=78.4, p<0.0001$, Fig. 2a), SG1OTU relative abundance was positively associated with chlorophyll $a(F=10.1, p=0.003$, Figs. $1 \mathrm{~b}$ and $2 \mathrm{~b})$ but negatively associated with surface salinity $(F=11.4, p=$ 0.002 , Fig. 2c), largely driven by recent sea-ice melt in the region.
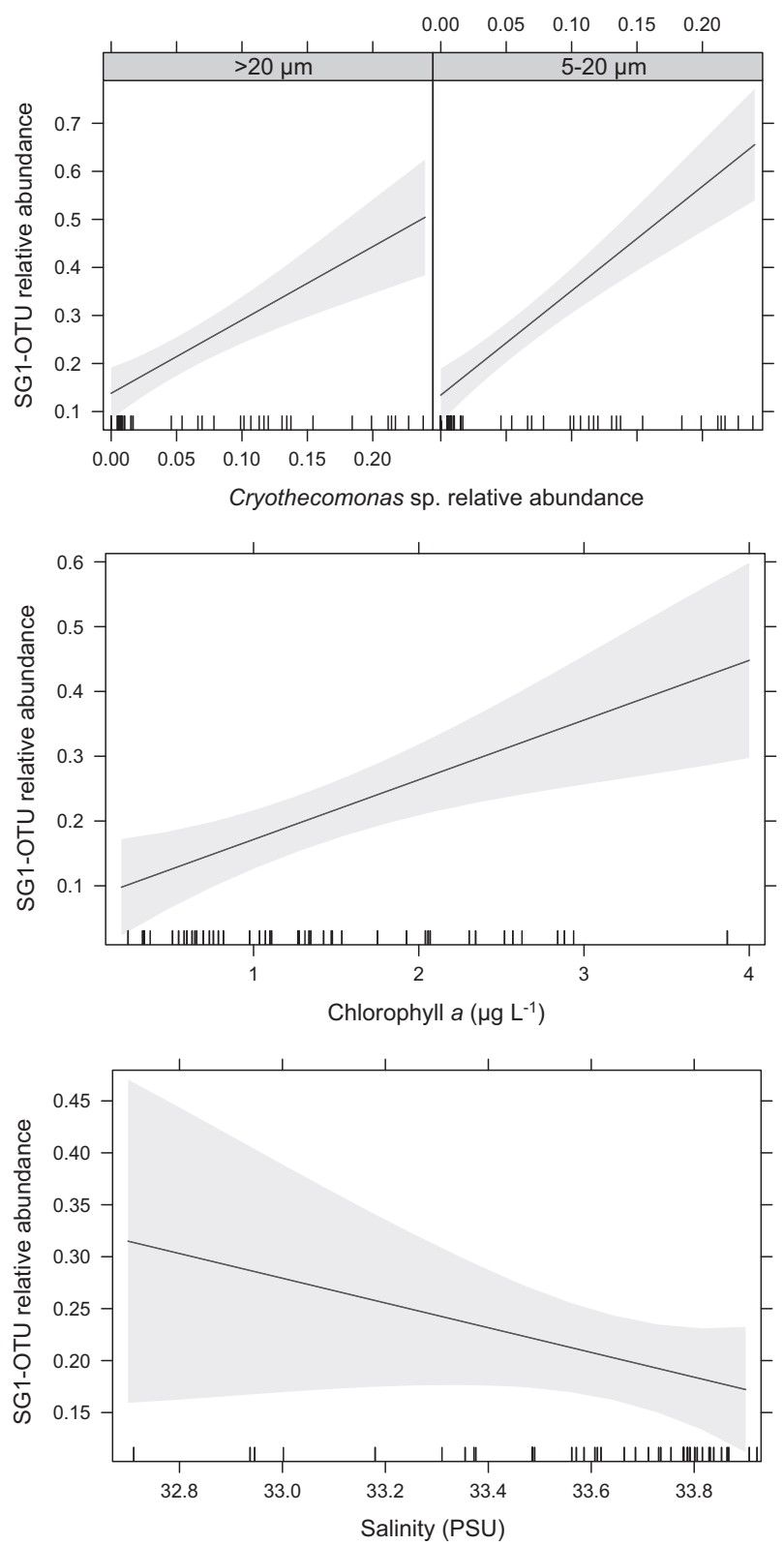

Fig. 2 Cryothecomonas OTU abundance and environment influence Syndiniales group I OTU abundance. Results of linear mixed effect model showing relationships between relative abundance of parasitic Syndiniales group I OTU, the Cryothecomonas sp. OTU, as well as chlorophyll $a$ and surface salinity. Model is based on the size fractions where the parasite is likely to be associated with the host (nanoplankton and microplankton). Relative abundances of OTUs were arcsine square-root transformed prior to modelling. See also Supplementary Figure 3 and Supplementary Table 1

\section{Discussion}

The abundance of Syndiniales, and particularly group I, in polar oceans including sea-ice habitats is increasingly being highlighted $[1,6,11]$. Our study shows that the SG1-OTU exhibits a global distribution, but represents $>20 \%$, and in some locations $>50 \%$, of eukaryote $18 \mathrm{~S}$ rDNA along Southern Ocean transects bounding an area of almost $190,000 \mathrm{~km}^{2}$ in the productive ice edge habitat.

We also report that Syndiniales group I parasites with identical 18S V4 sequences may associate with different hosts around the world. Closely related Syndiniales group I (clade 2) 18S V4 sequences (99.5\% identity) have been isolated from cercozoans and radiolarians ([12], Supplementary Figure 4). This suggests that either closely related Syndiniales parasites can infect distantly related hosts, or the SG1-OTU is a generalist that infects the most abundant rhizarian (cercozoan or radioloarian) host in a given environment. Alternatively, markers other than 18S rDNA may be required to differentiate between Syndiniales group I taxa. Designing fluorescent in situ hybridisation (FISH) probes for the SG1-OTU and putative hosts will help definitively establish parasite-host associations.

Our finding that chlorophyll $a$ was positively associated with SG1-OTU relative abundance, and improved models incorporating only host abundance, suggests higher parasite abundance in productive, high-biomass environments. Similarly, temporary domination of a North Pacific protist community by two parasitic (non-Syndiniales) taxa coincided with high chlorophyll $a$ concentrations immediately preceding collapse of the spring bloom [13]. Association of parasites with high-chlorophyll environments could be related to the greater chance of free-living spores encountering new hosts (higher infection rates); or that high chlorophyll areas represent productive environments, leading in some way to the production of more spores per host (higher reproduction rates [14]).

Despite the diversity and apparent ubiquity of Syndiniales in the world's oceans [1,2], parasites are rarely included in ecological/biogeochemical models [15, 16]. Parasites can alter ecosystem functioning and biodiversity through their influence on host traits and abundance [17]. As up to half the biomass of infected hosts may be converted to parasitoid biomass [7, 14], Syndiniales prevent or delay energy transfer to higher trophic levels. Parasitic Syndiniales, like viruses, cause some carbon and nutrients to be diverted to the microbial loop. Unlike viruses, Syndiniales dinospores are a potential food source for other protistan grazers such as tintinnids [7]. The availability of food web models incorporating parasitic interactions [16] argues for the inclusion of Syndiniales and other parasites in ecosystem models to better account for their role in carbon and nutrient cycling. 
Our findings indicate Syndiniales are key players in ice edge habitats. Given the importance of the sea ice to springsummer phytoplankton blooms and annual Southern Ocean primary productivity [18], and the potential for Syndiniales to impact both biological and geochemical processes, it is important we develop our understanding of their role in sea ice and other marine ecosystems.

\section{Data accessibility}

Sequences are available in GenBank under accession numbers MK002981-MK004953. OTU table, including contextual data are available on Figshare (https://doi.org/10. 6084/m9.figshare.7151510.v1). Chlorophyll data are available through the Australian Antarctic Data Centre [19].

Acknowledgements We thank the Australian Antarctic Division (AAD) Science Technical Support Team and Aurora Australis crew for making this work happen. Karen Westwood and Imojen Pearce (AAD) provided chlorophyll data. Ben Raymond and Mike Sumner (AAD) helped access and interpret sea-ice melt data. Ruth Eriksen (CSIRO), Andrea Polanowski, Andrew Davidson and Karen Westwood (AAD) provided assistance with lab work. Martin Ostrowski (Macquarie University) ran the OTU taxonomy assignment. Rowan Trebilco (ACE CRC) provided advice on analyses. Contribution to the Australian Antarctic Science Kerguelen Axis project (AAS-4344). Molecular work funded through the Australian Antarctic Science Program (AAS-4313) and a Bioplatforms Australia Industry Access Voucher. This work was supported by the Australian Government's Business Cooperative Research Centres Programme through the Antarctic Climate and Ecosystems Cooperative Research Centre, and the Australia Research Council's Special Research Initiative for Antarctic Gateway Partnership (Project ID SR140300001).

\section{Compliance with ethical standards}

Conflict of interest The authors declare that they have no conflict of interest.

\section{References}

1. de Vargas C, Audic S, Henry N, Decelle J, Mahé F, Logares R, et al. Eukaryotic plankton diversity in the sunlit ocean. Science. 2015;348:1261605.

2. Pernice MC, Giner CR, Logares R, Perera-Bel J, Acinas SG, Duarte CM, et al. Large variability of bathypelagic microbial eukaryotic communities across the world's oceans. ISME J. 2016;10:945-58.

3. Guillou L, Viprey M, Chambouvet A, Welsh RM, Kirkham AR, Massana R, et al. Widespread occurrence and genetic diversity of marine parasitoids belonging to Syndiniales (Alveolata). Environ Microbiol. 2008;10:3349-65.

4. Coats DW, Park MG. Parasitism of photosynthetic dinoflagellates by three strains of Amoebophrya (Dinophyta): Parasite survival, infectivity, generation time, and host specificity. J Phycol. 2002;38:520-8.

5. Chambouvet A, Morin P, Marie D, Guillou L. Control of toxic marine dinoflagellate blooms by serial parasitic killers. Science. 2008;322:1254-7.

6. Cleary AC, Durbin EG. Unexpected prevalence of parasite $18 \mathrm{~S}$ rDNA sequences in winter among Antarctic marine protists. J Plankton Res. 2016;38:401-17.

7. Salomon PS, Granéli E. Neves MHCB, Rodriguez EG. Infection by Amoebophrya spp. parasitoids of dinoflagellates in a tropical marine coastal area. Aquat Microb Ecol. 2009;55:143-53.

8. Eren AM, Morrison HG, Lescault PJ, Reveillaud J, Vineis JH, Sogin ML. Minimum entropy decomposition: unsupervised oligotyping for sensitive partitioning of high-throughput marker gene sequences. ISME J. 2015;9:968-79.

9. Bråte J, Krabberød AK, Dolven JK, Ose RF, Kristensen T, Bjørklund KR, et al. Radiolaria associated with large diversity of marine alveolates. Protist. 2012;163:767-77.

10. Thaler M, Lovejoy C. Distribution and diversity of a protist predator Cryothecomonas (Cercozoa) in Arctic marine waters. J Eukaryot Microbiol. 2012;59:291-9.

11. Bachy C, Lopez-Garcia P, Vereshchaka A, Moreira D. Diversity and vertical distribution of microbial eukaryotes in the snow, sea ice and seawater near the north pole at the end of the polar night. Front Microbiol. 2011;2:106.

12. Dolven JK, Lindqvist C, Albert VA, Bjørklund KR, Yuasa T, Takahashi $\mathrm{O}$, et al. Molecular diversity of alveolates associated with neritic North Atlantic radiolarians. Protist. 2007;158:65-76.

13. Berdjeb L, Parada A, Needham DM, Fuhrman JA. Short-term dynamics and interactions of marine protist communities during the spring-summer transition. ISME J. 2018: e-pub ahead of print 2018; https://doi.org/10.1038/s41396-018-0097-x.

14. Yih W, Coats DW. Infection of Gymnodinium sanguineum by the dinoflagellate Amoebophrya sp.: effect of nutrient environment on parasite generation time, reproduction, and infectivity. J Eukaryot Microbiol. 2000;47:504-10.

15. Worden AZ, Follows MJ, Giovannoni SJ, Wilken S, Zimmerman $\mathrm{AE}$, Keeling PJ. Environmental science. Rethinking the marine carbon cycle: factoring in the multifarious lifestyles of microbes. Science. 2015;347:1257594.

16. Warren CP, Pascual M, Lafferty KD, Kuris AM. The inverse niche model for food webs with parasites. Theor Ecol. 2010;3:285-94.

17. Frainer A, McKie BG, Amundsen PA, Knudsen R, Lafferty KD. Parasitism and the biodiversity-functioning relationship. Trends Ecol Evol. 2018;33:260-8.

18. Arrigo KR, van Dijken GL, Bushinsky S. Primary production in the Southern Ocean, 1997-2006. J Geophys Res. 2008;113: C08004 https://doi.org/10.1029/2007JC004551

19. Westwood K, Pearce I. Chlorophyll K-axis voyage V3 2015/16. Australian Antarctic Data Centre 2018. https://doi.org/10.4225/15/ $5 a 94 c 701 b 98 a 8$. 